\title{
Revealing hidden magnetoelectric multipoles using Compton scattering
}

\author{
Sayantika Bhowal $\odot$ and Nicola A. Spaldin $\odot$ \\ Materials Theory, ETH Zurich, Wolfgang-Pauli-Strasse 27, 8093 Zurich, Switzerland
}

(Received 5 May 2021; accepted 28 July 2021; published 23 August 2021)

\begin{abstract}
Magnetoelectric multipoles, which are odd under both space-inversion $\mathcal{I}$ and time-reversal $\mathcal{T}$ symmetries, are fundamental in understanding and characterizing magnetoelectric materials. However, the detection of these magnetoelectric multipoles is often not straightforward as they remain "hidden" in conventional experiments in part since many magnetoelectrics exhibit combined $\mathcal{I} \mathcal{T}$ symmetry. In this paper, we show that the antisymmetric Compton profile is a unique signature for all the magnetoelectric multipoles, since the asymmetric magnetization density of the magnetoelectric multipoles couples to space via spin-orbit coupling, resulting in an antisymmetric Compton profile. We develop the key physics of the antisymmetric Compton scattering using symmetry analysis and demonstrate it using explicit first-principles calculations for two well-known representative materials with magnetoelectric multipoles, insulating $\mathrm{LiNiPO}_{4}$ and metallic $\mathrm{Mn}_{2} \mathrm{Au}$. Our work emphasizes the crucial roles of the orientation of the spin moments, the spin-orbit coupling, and the band structure in generating the antisymmetric Compton profile in magnetoelectric materials.
\end{abstract}

DOI: 10.1103/PhysRevResearch.3.033185

\section{INTRODUCTION}

Multipoles provide a convenient basis for describing properties as diverse as electric charge densities and gravitational fields and are therefore widely used in many areas of physics, such as classical electromagnetism, metamaterials, and nuclear and particle physics [1-5]. They are particularly useful in condensed matter systems for characterizing the charge and spin and orbital magnetic moments of electrons in a unified way and have enabled understanding as well as prediction of various cross couplings and transport properties [6-9].

Multipoles can be categorized into four groups based on their behavior (odd or even) under time-reversal $(\mathcal{T}$ ) and space-inversion $(\mathcal{I})$ symmetries $[6,7,10]$. A substantial body of work in materials physics has focused on the evenparity (even under $\mathcal{I}$ ) multipoles, involving prediction and observation of hidden higher-order multipoles, beyond the conventional electric and magnetic dipoles, in the localized electrons of $d$ - and $f$-electron systems [11-13]. Recently, the search has extended to odd-parity multipoles, motivated in part by predictions of intriguing properties that may emerge from them $[6-9,14-16]$. The magnetoelectric (ME) multipoles, which are the subject of this work, form the lowest-order member of the family of multipoles that are odd under both space inversion and time reversal.

The ME multipole tensor, formally defined as $\mathcal{M}_{i j}=$ $\int r_{i} \mu_{j}(\vec{r}) d^{3} r$, describes the next order of spatial inhomogeneities in the magnetization density of a material, beyond

Published by the American Physical Society under the terms of the Creative Commons Attribution 4.0 International license. Further distribution of this work must maintain attribution to the author(s) and the published article's title, journal citation, and DOI. the magnetic dipole. By definition, the components of the tensor break both $\mathcal{I}$ and $\mathcal{T}$ symmetries, which is the same condition that allows the linear ME effect, in which an applied electric field induces a magnetization, $M_{i}=\alpha_{i j}^{\mathrm{ME}} E_{j}$, and vice versa. Indeed, the linear ME response of a material can be conveniently discussed in terms of the three irreducible (IR) components of the $\mathcal{M}_{i j}$ tensor, which are the scalar magnetoelectric monopole $a$, the vector toroidal moment $\vec{t}$, and the symmetric traceless quadrupole moment tensor $q_{i j}[6,8]$.

Direct experimental detection of these ME multipoles is a challenge in that they often remain "hidden" to conventional probes and analyses. To our knowledge to date, only resonant x-ray Bragg diffraction, nonreciprocal linear dichroism (magnetochiral dichroism), and polarized neutron diffraction have shown signatures of ME multipoles [17-20]. Recently, an additional possibility, Compton scattering, which measures the electron density as a function of momentum, was proposed as a candidate probe for the toroidal moment based on symmetry arguments relating a nonzero toroidal moment to an antisymmetric Compton profile [21]. While first-principles calculations indicated the required nonzero antisymmetric Compton scattering in two toroidal materials, $\mathrm{GaFeO}_{3}$ and $\mathrm{LiNiPO}_{4}$, preliminary experiments were unable to detect a signal above the noise level [21,22]. A detailed physical understanding of the process, however, which is crucial for identifying appropriate toroidal materials with stronger antisymmetric Compton responses, is still missing.

Here, we extend the earlier arguments of Ref. [21] to show that, in addition to the toroidal moment, the other ME multipoles, $a$ and $q_{i j}$, manifest in the antisymmetric Compton profile, vastly increasing the possible phase space of eligible materials. Using symmetry analysis, we extract the one-to-one correspondence between each of the ME multipoles and the direction in the momentum space along which the antisymmetric Compton profile appears. We then develop the key 
physics relating the antisymmetric Compton profile to the $\mathrm{ME}$ multipoles and show that the asymmetry in the magnetization density associated with the ME multipoles is mediated by the spin-orbit coupling (SOC) to the band structure asymmetry required for an antisymmetric Compton profile. Explicit first-principles calculations based on density functional theory (DFT) for two representative materials, insulating $\mathrm{LiNiPO}_{4}$ and metallic $\mathrm{Mn}_{2} \mathrm{Au}$, then allow us to determine the factorsband structure, fine details of the spin configuration, and SOC strength - that determine the strength of the antisymmetric Compton profile, suggesting guidelines for identifying materials with stronger effects.

Our work highlights the importance of the nontrivial duality between real and momentum space that causes the ME multipoles to be spin independent and of odd order in $k$ in momentum space. As a result, they can be detected in $k$ space using probes that are not spin resolved. In addition to the Compton scattering explored here, this observation implicates other momentum-space $k$-resolved but spin-insensitive probes, such as angle-resolved photoemission spectroscopy (ARPES), as suitable for direct observation of magnetoelectric multipoles.

\section{MAGNETOELECTRIC MULTIPOLES AND ANTISYMMETRIC COMPTON PROFILE}

We begin with a brief introduction to the ME multipoles (for a more detailed discussion, see, for example, Refs. [6,8]), with an emphasis on their manifestation in the antisymmetric Compton profile. Our focus is on the symmetry relations, as well as the nontrivial duality between the real and the momentum space, both of which are important in understanding the antisymmetric Compton profile.

\section{A. Magnetoelectric multipoles}

The ME multipoles, defined as $\mathcal{M}_{i j}=\int r_{i} \mu_{j}(\vec{r}) d^{3} r$, describe the inhomogeneity in the magnetization density $\vec{\mu}(\vec{r})$ (including both spin and orbital contributions), to lowest order beyond the usual magnetic dipole moment $\vec{m}=\int \vec{\mu}(\vec{r}) d^{3} r$. In contrast to the magnetic moment $\vec{m}$, which breaks only $\mathcal{T}$ symmetry, $\mathcal{M}_{i j}$ breaks both $\mathcal{I}$ and $\mathcal{T}$ symmetries as $\vec{r}$ and $\vec{\mu}$ break $\mathcal{I}$ and $\mathcal{T}$ symmetries, respectively. As a result, materials with nonzero $\mathcal{M}_{i j}$ satisfy the symmetry conditions required to show the linear ME effect, with a one-to-one correspondence between the matrix elements $\mathcal{M}_{i j}$ and the ME tensor elements $\alpha_{i j}^{\mathrm{ME}}$. The nine-component tensor $\mathcal{M}_{i j}$ can be decomposed into three IR components, the ME monopole $a$, toroidal moment $\vec{t}$, and ME quadrupole moment $q_{i j}$, such that

$\mathcal{M}=\left[\begin{array}{ccc}a+\frac{1}{2}\left(q_{x^{2}-y^{2}}-q_{z^{2}}\right) & t_{z}+q_{x y} & t_{y}+q_{x z} \\ -t_{z}+q_{x y} & a-\frac{1}{2} q_{x^{2}-y^{2}}-\frac{1}{2} q_{z^{2}} & -t_{x}+q_{y z} \\ -t_{y}+q_{x z} & t_{x}+q_{y z} & a+q_{z^{2}}\end{array}\right]$.

As seen from matrix (1), the ME monopole $a$ is a pseudoscalar (tensor of rank zero) and contributes to the diagonal
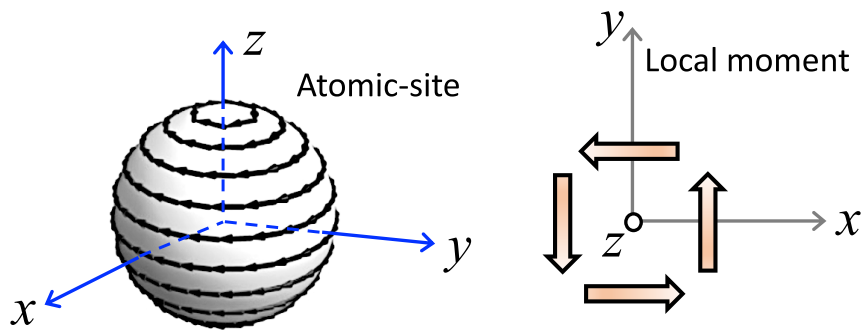

FIG. 1. Cartoons showing the atomic-site (as) and local-moment (loc) contributions to the ME multipole moments. The illustrations are for the $z$ component of the toroidal moment $t_{z}$. The magnetization texture on a sphere around an atom, as shown on the left, gives the as contribution to $t_{z}$. The loc contribution from the local magnetic dipole moments is shown on the right.

elements of the ME tensor,

$$
a=\frac{1}{3} \mathcal{M}_{i i}=\int \vec{r} \cdot \vec{\mu}(\vec{r}) d^{3} r,
$$

while the toroidal moment vector $\vec{t}$ is a tensor of rank 1 and constitutes the antisymmetric part of the $\mathcal{M}_{i j}$ tensor,

$$
t_{i}=\frac{1}{2} \varepsilon_{i j k} \mathcal{M}_{j k}=\frac{1}{2} \int \vec{r} \times \vec{\mu}(\vec{r}) d^{3} r .
$$

Finally, the five-component traceless quadrupole magnetic moment tensor $q_{i j}$ contributes to the symmetric part (both diagonal and off-diagonal elements) of the ME tensor,

$$
\begin{aligned}
q_{i j} & =\frac{1}{2}\left(\mathcal{M}_{i j}+\mathcal{M}_{j i}-\frac{2}{3} \delta_{i j} \mathcal{M}_{k k}\right) \\
& =\frac{1}{2} \int\left(r_{i} \mu_{j}+r_{j} \mu_{i}-\frac{2}{3} \delta_{i j} \vec{r} \cdot \vec{\mu}\right) d^{3} r .
\end{aligned}
$$

We point out that each of the ME multipoles in Eqs. (2)-(4) can be further decomposed into two contributions, one originating from the asymmetry in the magnetization density $\vec{\mu}(\vec{r})$ around an atomic site $\alpha$ [known as the atomic-site (as) contribution] and one from the asymmetric arrangement of the local dipole moments at the atomic sites, $\vec{m}_{\alpha}$, produced from the same magnetization density $\vec{\mu}(\vec{r})$ [called the local-moment (loc) contribution] [8]. The two contributions are illustrated schematically for the case of the $t_{z}$ component of the toroidal moment in Fig. 1. Mathematically, the decomposition for the toroidal moment is given by [23]

$$
\begin{aligned}
\vec{t} & =2^{-1} \int \vec{r} \times \vec{\mu}(\vec{r}) d^{3} r \\
& =2^{-1} \sum_{\alpha} \int_{a s}\left[\left(\vec{r}-\vec{r}_{\alpha}\right) \times \mu(\vec{r})+\vec{r}_{\alpha} \times \vec{\mu}(\vec{r})\right] d^{3} r \\
& =2^{-1} \sum_{\alpha} \int_{a s}\left(\vec{r}-\vec{r}_{\alpha}\right) \times \mu(\vec{r}) d^{3} r+2^{-1} \sum_{\alpha} \vec{r}_{\alpha} \times \vec{m}_{\alpha} \\
& =\vec{t}_{\text {as }}+\vec{t}_{\mathrm{loc}},
\end{aligned}
$$

where the summation is over all the atoms $\alpha$ that have a local magnetic moment. The integral in the as contribution, the first term of Eq. (5), is over a localized region around the atomic nucleus at $\vec{r}_{\alpha}$. In an electronic structure calculation, the 
integral can be performed within an atomic sphere or muffin tin of a chosen radius around the nucleus.

\section{B. Antisymmetric Compton profile}

Next we show that all the ME multipoles described above contribute to the antisymmetric Compton profile and identify the correspondence between each specific ME multipole and the antisymmetric Compton profile along a particular direction in momentum space.

Compton scattering is an inelastic $\mathrm{x}$-ray scattering process in which the energy loss of the photon is proportional to the projection of the electron momentum density along the direction of the photon momentum transfer. As a result, the measured double-differential cross section $\frac{d^{2} \sigma}{d \Omega d E}$ along direction $z$, say, is linearly proportional to the Compton scattering profile $J\left(p_{z}\right)$, which is related to the electron momentum density $\rho(\vec{p})$ by

$$
J\left(p_{z}\right)=\int \rho(\vec{p}) d p_{x} d p_{y} .
$$

In materials where either of the two symmetries, space inversion or time reversal, is present or both are present, $\rho(\vec{p})=$ $\rho(-\vec{p})$, and the Compton scattering profile is symmetric in momentum. If both symmetries are broken, however, neither the electron momentum density $\rho(\vec{p})$ nor the Compton profile $J\left(p_{z}\right)$ are required to be symmetric with respect to $\vec{p}$. This was pointed out in Ref. [21], where it was suggested that Compton scattering could therefore be used to probe the toroidal moment $\vec{t}$. Here, we extend Ref. [21] to show that not only the toroidal moments but also all the other ME multipoles can have an antisymmetric contribution to their Compton scattering.

Next we connect the Compton profile to the momentumspace representation of the ME multipoles via the multipole expansion of the density matrix. The parity-odd and timeodd sector of the density matrix is of interest to us, as it corresponds to the antisymmetric part of the Compton profile. Therefore we expand the density matrix $[\rho]$, with respect to its behavior under space inversion and time reversal,

$$
\begin{aligned}
{[\rho] } & =\sum_{\nu=0,1} \sum_{\eta=0,1} \rho^{\nu \eta}, \\
\text { where } \mathcal{T} \rho^{\nu \eta} & =(-1)^{\nu} \rho^{\nu \eta}, \quad \mathcal{I} \rho^{\nu \eta}=(-1)^{\eta} \rho^{\nu \eta} .
\end{aligned}
$$

Expanding the part of the density matrix that is odd under both space and time inversion, $\rho^{11}$, in terms of the ME multipoles in Eqs. (2)-(4), we obtain [8]

$$
\rho^{11}=\sum_{\gamma, \beta} \Gamma_{\beta}^{11 \gamma} T_{\beta}^{\gamma}
$$

Here, $\gamma=|v-\eta|, \ldots, v+\eta=0,1,2$, represents the rank of the parity-odd $(\eta=1)$ and time-odd $(v=1)$ ME multipoles, discussed in Sec. II A, with $\gamma=0,1,2$ corresponding to the ME monopole, the toroidal moment, and the quadrupole moment, respectively, and the integer $\beta$ runs from $-\gamma$ to $\gamma$. Since $\rho^{11}$ describes the component of the density that is odd under both time reversal and space inversion, we can conclude that all ME multipoles contribute to the antisymmetric Compton profile.
Next we analyze the momentum-space representations of the time-odd, parity-odd multipoles, in order to establish the directions in which the antisymmetric Compton profile is nonzero. The momentum-space representations of the oddparity multipoles have a different form from their real-space representations due to the nontrivial duality between the real space and the momentum space. We can see this from the following considerations: The functional form of the spatial part of the odd-parity multipoles must be of odd order in both $r$ and $k$ space, because both $r$ and $k$ change sign under inversion, i.e., $\vec{r} \stackrel{\mathcal{I}}{\rightarrow}-\vec{r}$ and $\vec{k} \stackrel{\mathcal{I}}{\rightarrow}-\vec{k}$. Under time reversal, however, the behavior of $r$ and $k$ is different, with $\vec{r} \stackrel{\mathcal{T}}{\rightarrow} \vec{r}$ but $\vec{k} \stackrel{\mathcal{T}}{\rightarrow}-\vec{k}$. As a result, the spin dependence must be different in the real-space and the momentum-space representations. This leads to the following intriguing consequences for the oddparity multipoles: First, the basis functions of the odd-parity magnetic multipoles in momentum space are spin independent but of odd order in $k$. This gives rise to antisymmetric electron (and magnon) dispersions [15,24], which manifest as the antisymmetric Compton profile. Second, the specific basis function of the allowed ME multipole in a system dictates the momentum direction of the antisymmetric Compton profile. The detection of the antisymmetric Compton profile along a certain direction, therefore, can be used to identify the presence of a particular ME multipole in a material.

\section{ANTISYMMETRIC COMPTON PROFILE IN EXAMPLE SYSTEMS}

In order to illustrate the above ideas of the antisymmetric Compton profile, we explicitly compute and analyze the Compton profile, for two example materials (i) $\mathrm{LiNiPO}_{4}$ and (ii) $\mathrm{Mn}_{2} \mathrm{Au}$. While $\mathrm{LiNiPO}_{4}$ is an insulating system and exhibits the linear $\mathrm{ME}$ effect [25], $\mathrm{Mn}_{2} \mathrm{Au}$ is an antiferromagnetic (AFM) metal, in which an electric current has been shown to reorient the direction of the magnetization [26-29]. The presence of ME multipoles has been demonstrated computationally for both materials, and their relevance for the electric-field- or electric-current-induced magnetism has been discussed [8,29]. Here, using a combination of symmetry analysis and DFT calculations, we show that the antisymmetric Compton profile acts as a unique fingerprint of the symmetry-allowed ME multipoles in these materials. From a detailed analysis of the DFT results, we extract the role of the magnetic structure, band structure, and SOC effects in the antisymmetric Compton scattering. The insight into the physical mechanism that these provide is a first step toward identifying materials with a larger antisymmetric Compton response.

\section{A. Insulating system: $\mathrm{LiNiPO}_{4}$}

$\mathrm{LiNiPO}_{4}$ crystallizes in the olivine structure with the orthorhombic space group Pnma and the crystallographic point group $D_{2 h}$ [30]. The crystal structure, in which the $\mathrm{Ni}$ atoms at Wyckoff positions $4 c$ are surrounded by six oxygen atoms forming distorted $\mathrm{NiO}_{6}$ octahedra, is shown in Fig. 2(a). The material undergoes various magnetic transitions in the presence of a magnetic field, and the resulting phases exhibit linear 

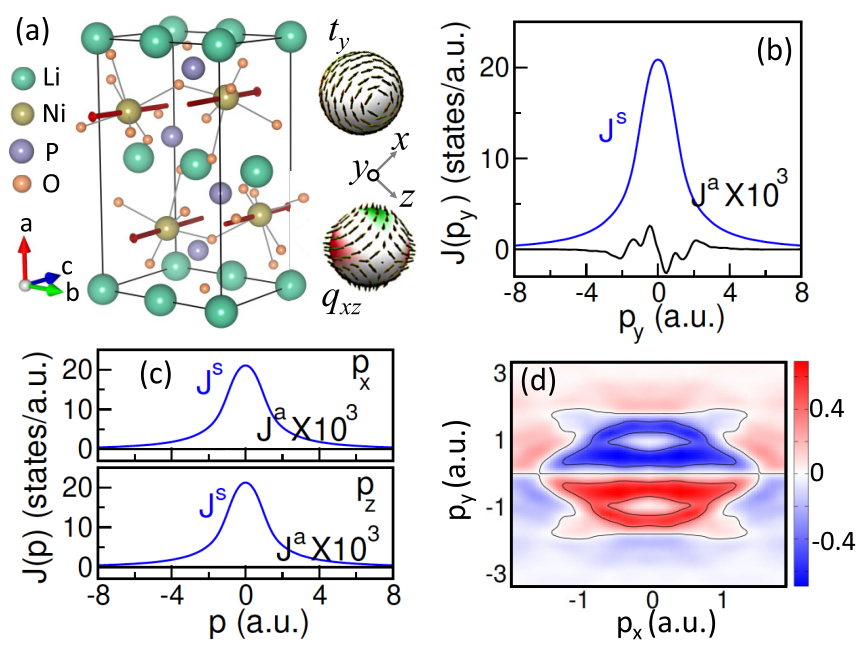

FIG. 2. Antisymmetric Compton profile in $\mathrm{LiNiPO}_{4}$. (a) The crystal and magnetic $\left(P n m^{\prime} a\right)$ structures of $\mathrm{LiNiPO}_{4}$. The arrows indicate the spin directions at the $\mathrm{Ni}$ sites. Representation of the allowed ME multipoles, toroidal moment $t_{y}$ and quadrupole moment $q_{x z}$, in this magnetic structure are shown at the right. (b) The symmetric $\left(J^{s}\right)$ and the antisymmetric $\left(J^{a}\right)$ parts of the Compton profile along the $y$ direction in the momentum space, computed for the magnetic structure, shown in (a). (c) The absence of the antisymmetric part for other momentum directions, $p_{x}$ and $p_{z}$. (d) The distribution of the antisymmetric part of the line integral of the electron momentum density along $p_{z}$ in the $p_{x}-p_{y}$ plane of the momentum space, indicating, further, the antisymmetry along $p_{y}$. The antisymmetric parts in all figures are magnified by a factor of $10^{3}$.

and quadratic ME effects [31]. Out of these various magnetic phases, the magnetic structure corresponding to the magnetic space group Pnm'a with the propagation vector $\vec{q}=(0,0,0)$ constitutes the magnetic ground state of the system. In this magnetic structure, the $\mathrm{Ni}$ atoms are antiferromagnetically ordered, with the major spin component along the $z$ direction and a small $x$ component due to canting [see Fig. 2(a)]. The magnetic configuration breaks the inversion symmetry of the structure, allowing for ME toroidal and quadrupole moments consistent with its off-diagonal linear ME effect [8,31,32]. Here, we discuss the manifestation of these allowed ME multipoles in the antisymmetric Compton profile of $\mathrm{LiNiPO}_{4}$.

\section{Symmetry analysis}

Before presenting the results of DFT calculations for the antisymmetric Compton profile in $\mathrm{LiNiPO}_{4}$, we first analyze the symmetry of the magnetic structure and the allowed ME multipoles. This symmetry analysis reveals the direction(s) of the nonzero antisymmetric Compton profile in momentum space, which we further verify in our DFT calculations presented in Sec. III A 2.

The real- and momentum-space basis functions for the time-odd, parity-odd ME multipoles, corresponding to the point group $D_{2 h}$, of $\mathrm{LiNiPO}_{4}$, are obtained following Ref. [24], and using the following compatibility relations [33,34], the IRs of the $D_{4 h}$ point group reduce to the $D_{2 h}$ point group as $A_{1 u} \downarrow D_{2 h}=A_{u}, B_{1 u} \downarrow D_{2 h}=A_{u}, A_{2 u} \downarrow D_{2 h}=B_{1 u}$,
$B_{2 u} \downarrow D_{2 h}=B_{1 u}$, and $E_{u} \downarrow D_{2 h}=B_{2 u}+B_{3 u}$. The results are listed in Table I.

The magnetic ground state $\left(P n m^{\prime} a\right)$ of $\mathrm{LiNiPO}_{4}$ corresponds to the IR representation $B_{2 u}^{-}$. As seen from Table I, this $B_{2 u}^{-}$representation allows for a net toroidal moment along the $y$ direction $\left(t_{y}\right)$ and a $q_{x z}$ quadrupole moment. The magnetic point group $\mathrm{mm}^{\prime} \mathrm{m}$ at the $\mathrm{Ni}$ site allows, in addition, for the atomic-site ME monopole $a$ and quadrupole moments $q_{x^{2}-y^{2}}$ and $q_{z^{2}}$. These, however, are arranged in an antiferro-type pattern between the $\mathrm{Ni}$ atoms, so that the net values of these ME multipoles are zero.

We now focus on the basis functions for these ME multipoles, which is crucial for the desired antisymmetric Compton profile. As expected from the discussion of the real-spacemomentum-space duality in Sec. II B, the real-space basis functions in Table I are spin dependent while the momentumspace basis functions are not. More interestingly, the basis functions in the momentum space are always of odd order in $k$. For example, the momentum-space basis for the ME multipoles $t_{y}, q_{x z}$ (in the $B_{2 u}^{-}$representation) is $k_{y}$, indicating an asymmetric dispersion along the $k_{y}$ direction. Thus, for $\mathrm{LiNiPO}_{4}$, we expect an antisymmetric Compton profile along the $y$ direction in momentum space, as well as along any directions with $k_{y} \neq 0$.

It is interesting to point out here that each of the ME multipoles in Table I allows for an antisymmetric Compton profile. For example, the existence of the ME multipoles $\left\{t_{x}, q_{y z}\right\}$ and $\left\{t_{z}, q_{x y}\right\}$ indicates an antisymmetric profile along $k_{x}$ (and any direction with $k_{x} \neq 0$ ) and $k_{z}$ (and any direction with $k_{z} \neq 0$ ), respectively. The situation is, however, different for the $\mathrm{ME}$ monopole moment $a$ and the quadrupole moments $q_{x^{2}-y^{2}}$ and $q_{z^{2}}$, which give rise to an antisymmetric profile only along the direction in the momentum space, where simultaneously the $k_{x} \neq 0, k_{y} \neq 0$, and $k_{z} \neq 0$ conditions are satisfied (see Table I). While these symmetry arguments are useful for predicting the directions in which an antisymmetric Compton profile will occur, to develop an understanding of the process and, in particular, to identify materials with large responses, it is instructive to know the magnitude of the antisymmetric profile.

\section{DFT results for the Compton profile and atomic-site multipoles}

In order to verify the above symmetry analysis as well as to gain insight into the physics of the antisymmetric Compton profile, we next compute the antisymmetric Compton profile for $\mathrm{LiNiPO}_{4}$ using the linearized augmented plane wave (LAPW) method as implemented in the ELK code $[35,36]$. We use the local-density approximation (LDA) $+\mathrm{SOC}+U$ formalism, with $U=5 \mathrm{eV}$ and $J=0.75 \mathrm{eV}$ at the Ni site, except in the cases for which we study the effects of varying these parameters. A basis set of $l_{\max (\mathrm{apw})}=8$ and a $3 \times 6 \times 6 \mathrm{k}$-point sampling of the Brillouin zone (BZ) are used to achieve selfconsistency. The product of the muffin-tin radius (2.0, 2.4, 2.2, and 1.8 a.u. for $\mathrm{Li}, \mathrm{Ni}, \mathrm{P}$, and $\mathrm{O}$, respectively) and the maximum reciprocal lattice vector is taken to be 7 . All calculations are done at the relaxed atomic positions and lattice constants reported in Ref. [8]. The electron momentum densities are calculated and projected onto the selected momentum directions $(\vec{p})$ to obtain the Compton profile $J(\vec{p})$ [36]. The computed 
TABLE I. The basis functions for the ME multipoles for the $D_{2 h}$ point group: ME monopole $a$, toroidal moment $\vec{t}$, and quadrupole moment $q_{i j}$. Here, $m_{x}, m_{y}$, and $m_{z}$ are the $x, y$, and $z$ components of the magnetic moment $\vec{m}$, which includes both spin and orbital contributions, $\vec{m} \equiv \mu_{B}\left(\frac{2 \vec{l}}{l+1}+2 \vec{s}\right) \cdot \vec{l}$ and $\vec{s}$ are the orbital and the spin angular momentum, respectively.

\begin{tabular}{lccc}
\hline \hline IR & & & Basis \\
& ME multipole & Real space & $k$ space \\
\hline$A_{u}^{-}$ & $a$, & $x m_{x}+y m_{y}+z m_{z}$, & $k_{x} k_{y} k_{z}$ \\
& $q_{x^{2}-y^{2}}$ & $\left(x m_{x}-y m_{y}\right)$, & \\
$B_{1 u}^{-}$ & $q_{z^{2}}$ & $\left(2 z m_{z}-x m_{x}-y m_{y}\right)$ & $k_{z}$ \\
$B_{2 u}^{-}$ & $t_{z}, q_{x y}$ & $\left(x m_{y}-y m_{x}\right),\left(x m_{y}+y m_{x}\right)$ & $k_{y}$ \\
$B_{3 u}^{-}$ & $t_{y}, q_{x z}$ & $\left(z m_{x}-x m_{z}\right),\left(z m_{x}+x m_{z}\right)$ & $k_{x}$ \\
\hline \hline
\end{tabular}

profile is, further, separated into symmetric $J^{s}(\vec{p})$ and antisymmetric $J^{a}(\vec{p})$ parts, using $J(\vec{p})=2^{-1}[J(\vec{p})+J(-\vec{p})]+$ $2^{-1}[J(\vec{p})-J(-\vec{p})]=J^{s}(\vec{p})+J^{a}(\vec{p})$. The convergence of the symmetric and the antisymmetric parts of the profile is confirmed by performing additional calculations with the denser $5 \times 10 \times 10 k$-point mesh. The computed symmetric and antisymmetric parts of the profiles are normalized to the number of valence electrons per formula unit of $\mathrm{LiNiPO}_{4}$ in the calculation, which is 48 electrons (see Ref. [37]). The isotropic core contribution, obtained from the Hartree-Fock calculations of Biggs et al. [38], is further added to the symmetric part of the profile to obtain the total Compton profile.

Our computed Compton profiles for the magnetic ground state along the crystallographic $x, y$, and $z$ directions [Figs. 2(b) and 2(c)] show that the antisymmetric part $J^{a}(\vec{p})$ is only nonzero along the $y$ direction in momentum space, consistent with the symmetry analysis. The symmetric and the antisymmetric parts of the profile along $p_{y}$ are shown in Fig. 2(b). As seen from this figure, both $J^{s}\left(p_{y}\right)$ and $J^{a}\left(p_{y}\right)$ satisfy the zero-sum rule $\int_{-\infty}^{\infty} p_{y} J^{s, a}\left(p_{y}\right) d p_{y}=0$. While this sum rule is trivial for the symmetric part, it imposes rather a stringent constraint on each half of the antisymmetric profile, which is $\int_{0}^{\infty} p_{y} J^{a}\left(p_{y}\right) d p_{y}=0$ [21]. This condition indicates that for each half of $J^{a}\left(p_{y}\right)$, a positive contribution is always accompanied by a negative contribution, and vice versa, which can also be verified visually from Fig. 2(b). Note that the computed antisymmetric part is rather small in magnitude, at least three orders of magnitude smaller than that of $J^{s}\left(p_{y}\right)$ and extended beyond 4 a.u. of momentum, similar to the antisymmetric Compton profile in $\mathrm{GaFeO}_{3}$ reported earlier [21]. The antisymmetric part of the line integral of the electron momentum density $\int \rho^{a}(\vec{p}) d p_{z}$ in the $p_{x}-p_{y}$ plane is shown in Fig. 2(d), where it is evident that this quantity is antisymmetric along $p_{y}$.

The obtained antisymmetric Compton profile can be related to the details of the band structure as follows: The presence or absence of $\mathcal{I}$ and $\mathcal{T}$ symmetries dictates the symmetry conditions on the band energies $E$ for any arbitrary momentum point $\vec{k}$ and the corresponding $-\vec{k}$, with $E(\vec{k} \uparrow) \stackrel{\mathcal{I}}{\rightarrow} E(-\vec{k} \uparrow)$, while $E(\vec{k} \uparrow) \stackrel{\mathcal{T}}{\rightarrow} E(-\vec{k} \downarrow)$. The combination of both $\mathcal{I}$ and $\mathcal{T}$ symmetries consequently leads to doubly degenerate bands at every momentum point in the BZ, $E(\vec{k} \uparrow) \stackrel{\mathcal{I} \mathcal{T}}{\longrightarrow} E(\vec{k} \downarrow)$. In the present case, individual $\mathcal{I}$ and $\mathcal{T}$ symmetries are broken, but the combined $\mathcal{I} \mathcal{T}$ symmetry is preserved. In this case, all bands remain doubly degenerate everywhere in the BZ due to the $\mathcal{I} \mathcal{T}$ symmetry; however, there is no symmetry restriction, as the individual $\mathcal{I}$ and $\mathcal{T}$ symmetries are broken, that guarantees that bands at $\vec{k}$ and $-\vec{k}$ have the same energies. This leads to an asymmetric band structure in momentum space.

The direction of the asymmetric band structure is determined by the momentum-space basis function of the specific parity-odd, time-odd multipoles, present in the system. In the case of $\mathrm{LiNiPO}_{4}$, the ME multipoles $t_{y}$ and $q_{x z}$ give rise to an asymmetric band structure along $k_{y}$, as shown in the left panel of Fig. 3(a). This asymmetric band structure, further, leads to the asymmetric Compton profile. Therefore the asymmetry in the Compton profile, determined by the antisymmetric part $J^{a}$, is a measure of the parity-odd, time-odd ME multipoles.

It is important to point out, here, that the SOC is absolutely essential to create such an asymmetric band structure and, hence, the asymmetric Compton profile. This is because the asymmetric magnetization density due to broken $\mathcal{I}$ and $\mathcal{T}$ symmetries, which is represented by the ME multipoles, can only couple to the space via SOC. In the absence of SOC, such coupling is absent, and the band structure remains symmetric as shown in the right panel of Fig. 3(a). Since the antisymmetric Compton profile is a manifestation of such an asymmetric band structure, it in turn also occurs only in the presence of the SOC. In fact, the magnitude of $J^{a}\left(p_{y}\right)$ has a strong dependence on the strength of the SOC, as we can see by artificially changing the $\mathrm{SOC}$ value in $\mathrm{LiNiPO}_{4}$. As depicted in Fig. 3(b), we find that the antisymmetric Compton profile decreases substantially as we decrease the strength of the SOC constant, $\lambda$.

On the other hand, the dependence of $J^{a}\left(p_{y}\right)$ on the value of the Coulomb interaction parameter $U$ is quite weak, with the height of the peak in $J^{a}\left(p_{y}\right)$ decreasing only slightly with increasing $U$ [see Fig. 3(c)]. To understand the weak $U$ dependence, we next calculate the $U$ dependence of the symmetry-allowed ME multipoles on the $\mathrm{Ni}$ ions. As described above, the atomic-site multipoles are extracted by decomposing the density matrix $\rho_{l m, l^{\prime} m^{\prime}}$ into its tensor moments, of which the parity-odd tensor moments have contributions only from the odd $l-l^{\prime}$ terms [8]. We therefore evaluate both the $p-d$ and $p-s$ matrix element contributions, using the ELK code, with the computational parameters described above. In this density matrix decomposition, $\gamma=1$ 

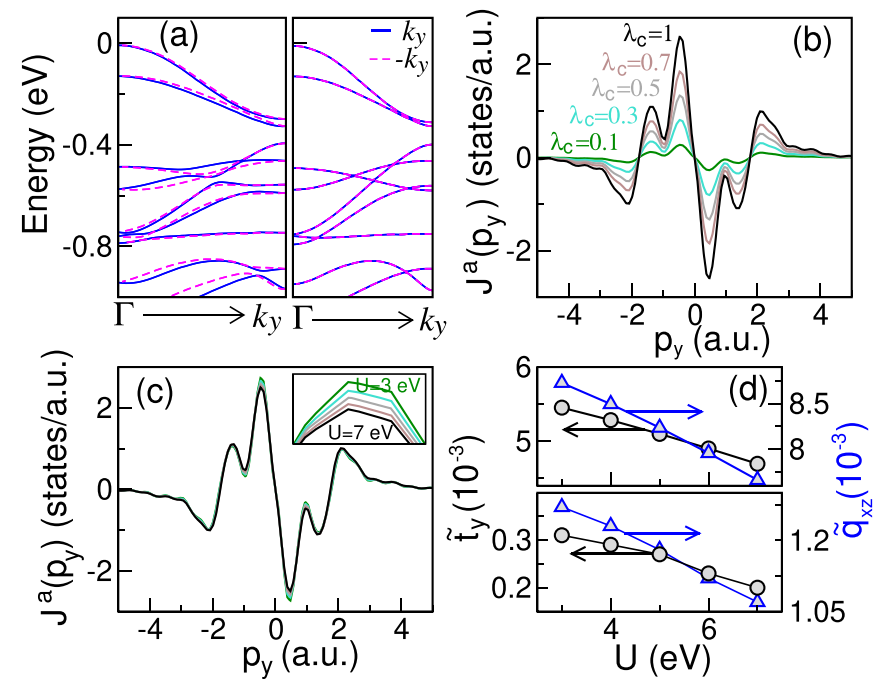

FIG. 3. Role of SOC in the antisymmetric Compton profile. (a) Band structure along $k_{y}$ for the magnetic configuration in Fig. 2(a) both in the presence (left) and absence (right) of SOC. As seen from this plot, the bands are symmetric in the absence of SOC, while the presence of asymmetry is visible when SOC effects are included, indicating the crucial role of SOC in generating the asymmetry in the band dispersion. This is also reflected in the antisymmetric Compton profile $J^{a}\left(p_{y}\right)$ in (b). (b) The variation of $J^{a}\left(p_{y}\right)$ as a function of SOC, indicating strong dependence on the scaled SOC $\lambda=\lambda_{c} \times \lambda_{r}$, where $\lambda_{r}$ is the strength of the SOC in the real material and $\lambda$ is the SOC that we artificially enforce in the calculation. (c) The variation of $J^{a}\left(p_{y}\right)$ with the Hubbard $U$, indicating a weak dependence on $U$. The inset shows the small changes near the peak for a $U$ variation of 3-7 eV in intervals of $1 \mathrm{eV}$. (d) The variation of the magnitude of the computed atomic-site ME multipoles $\tilde{t}_{y}$ and $\tilde{q}_{x z}$ (in units of $\mu_{B}$ ) with $U$ (top panel). As in (c), both decrease with increasing $U$. The same variation for a different muffin-tin radius (2.6 a.u.) of $\mathrm{Ni}$ (bottom panel). Although the magnitudes of the multipoles depend on the muffin-tin radius, the trend in their variations with $U$ remains the same.

and $\beta=-1$ then provides a measure of $t_{y}$, while $\gamma=2$ and $\beta=1$ gives a measure of the $q_{x z}$ multipole. We label these quantities $\tilde{t}_{y}$ and $\tilde{q}_{x z}$ in the following and note that their units are $\mu_{B}$. We see that the weak decrease in the Compton profile on increasing $U$ may be attributed to the slight decrease in the computed value of the ME multipoles $\tilde{t}_{y}$ and $\tilde{q}_{x z}$ as $U$ increases, shown in the top panel of Fig. 3(d). Note that the explicit value of the atomic-site contribution to the $\mathrm{ME}$ multipole depends on the choice of atomic muffin-tin radius; however, the trend in the values remains unchanged [see, e.g., the bottom panel of Fig. 3(d)].

\section{Role of ME multipoles: Effect of manipulation of the magnetic structure}

To further elucidate the role of the ME multipoles in the Compton profile, we next explicitly compute how changes in the local magnetic dipolar order change both the multipoles and the Compton profile. In particular, we manipulate the local magnetic dipolar order of the system, to either cause a change in the magnitude of the multipoles or give rise to
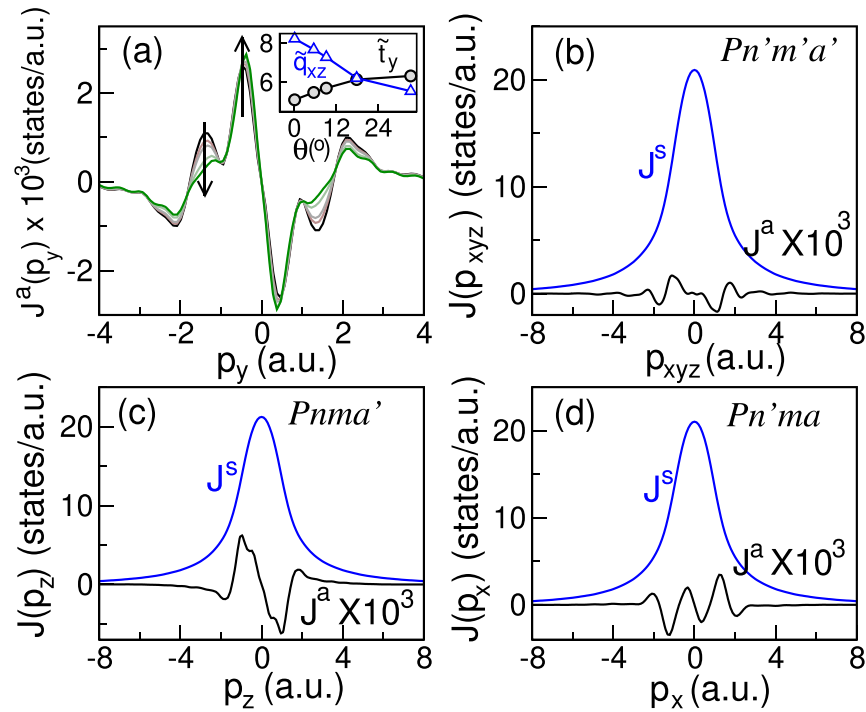

FIG. 4. Manipulation of the magnetic structure and the corresponding antisymmetric Compton profile. (a) The variation of the antisymmetric Compton profile as a function of canting angle $\theta$ from $0^{\circ}$ to $33^{\circ}$. The arrows indicate the direction of increasing values of the canting angle $\theta$. The inset shows the corresponding variation in the magnitudes of the computed atomic-site ME toroidal moment $\tilde{t}_{y}$ and the quadrupole moment $\tilde{q}_{x z}$ in units of $10^{-3} \mu_{B}$ for the same values of $\theta$ as in the main plot. (b)-(d) The symmetric $\left(J^{s}\right)$ and the antisymmetric $\left(J^{a}\right)$ parts of the Compton profile along (b) the $p_{x y z}$ direction for the magnetic structure $P n^{\prime} m^{\prime} a^{\prime}$, (c) the $p_{z}$ direction for the magnetic space group $P n m a^{\prime}$, and (d) the $p_{x}$ direction for the magnetic space group $P n^{\prime} m a$. In all cases the antisymmetric part of the Compton profile is magnified by a factor of $10^{3}$.

an entirely new set of ME multipoles. We then calculate the corresponding changes in the antisymmetric Compton profile. We compute the changes in both the atomic-site and localmoment contributions.

In the Pnm'a magnetic ground state of $\mathrm{LiNiPO}_{4}$, the spin moments are primarily oriented along the $z$ direction $\left(m_{z}\right)$ with a small canting angle $\theta=\tan ^{-1}\left(m_{x} / m_{z}\right)$, which gives rise to a tiny $x$ component of moment $\left(m_{x}\right)$ as well [32]. We investigate the effect of changing this canting angle $\theta$ on both the ME multipoles and the Compton profile. While changing $\theta$, the spins are always kept in the $x-z$ plane, and the $P n m^{\prime}$ a magnetic symmetry of the structure is also preserved. Since this particular magnetic symmetry allows ferro-type ordering for only $t_{y}$ and $q_{x z}$, no additional ferro-type ME multipoles appear as a result of change in the canting angle, but the magnitude of these multipoles may vary with the change in $\theta$. Indeed, with increase in $\theta$ the magnitude of $\tilde{t}_{y}$ increases, while that of $\tilde{q}_{x z}$ decreases, as shown in the inset of Fig. 4(a).

Similar behavior is also seen in the local-moment (loc) contributions to $t_{y}$ and $q_{x z}$, where the loc contribution to $\vec{t}, \vec{t}_{\mathrm{loc}}$, can be calculated using Eq. (5), and the quadrupole moment can be calculated similarly using $\left(q_{x z}\right)_{\text {loc }}=2^{-1} \sum_{\alpha}\left(x^{\alpha} m_{z}^{\alpha}+\right.$ $\left.z^{\alpha} m_{x}^{\alpha}\right)$. Here, $x^{\alpha}$ and $z^{\alpha}$ denote the $x$ and $z$ Cartesian coordinates of the $\mathrm{Ni}$ atom at site $\alpha$, and $m_{x}^{\alpha}$ and $m_{z}^{\alpha}$ are the corresponding Cartesian components of the spin moments, as listed in Table II. Performing straightforward algebra, we 
TABLE II. The coordinates $\left(x^{\alpha}, y^{\alpha}, z^{\alpha}\right)$ and the corresponding spin moment direction $\left(m_{x}^{\alpha}, m_{y}^{\alpha}, m_{z}^{\alpha}\right)$ at each Ni site $\alpha$ corresponding to the Wyckoff positions $4 c$ of the crystallographic space group Pnma and the magnetic space group Pnm'a. $\epsilon$ and $\delta$ are the internal structural parameters for $\mathrm{LiNiPO}_{4}$ with orthorhombic lattice constants $a, b$, and $c . S$ and $\theta$ are the magnitude of the Ni spin moment and the canting angle, respectively.

\begin{tabular}{|c|c|c|c|c|c|c|}
\hline $\operatorname{Site}(\alpha)$ & $x^{\alpha} / a$ & $y^{\alpha} / b$ & $z^{\alpha} / c$ & $m_{x}^{\alpha}$ & $m_{y}^{\alpha}$ & $m_{z}^{\alpha}$ \\
\hline $\mathrm{Ni}(1)$ & $-\epsilon+1 / 2$ & $3 / 4$ & $\delta+1 / 2$ & $S \sin \theta$ & 0 & $S \cos \theta$ \\
\hline $\mathrm{Ni}(2)$ & $\epsilon$ & $1 / 4$ & $\delta$ & $S \sin \theta$ & 0 & $-S \cos \theta$ \\
\hline $\mathrm{Ni}(3)$ & $\epsilon+1 / 2$ & $1 / 4$ & $-\delta+1 / 2$ & $-S \sin \theta$ & 0 & $-S \cos \theta$ \\
\hline $\mathrm{Ni}(4)$ & $-\epsilon$ & $3 / 4$ & $-\delta$ & $-S \sin \theta$ & 0 & $S \cos \theta$ \\
\hline
\end{tabular}

obtain the local-moment contribution to $\vec{t}$,

$$
\vec{t}_{\mathrm{loc}}=\frac{S}{2}\left[\sin \theta\left(\begin{array}{c}
0 \\
4 \delta c+r c \\
q b
\end{array}\right)+\cos \theta\left(\begin{array}{c}
q b \\
4 \epsilon a+p a \\
0
\end{array}\right)\right] .
$$

Here, $a, b$, and $c$ are the orthorhombic lattice constants, $p$, $q$, and $r$ are arbitrary integers, and $S$ is the magnitude of the $\mathrm{Ni}$ spin moment. It is easy to see that a nontrivial $\vec{t}_{\text {loc }}$ exists only along the $y$ direction, as it should according to symmetry, and that this $y$ component increases with increasing $\theta$. In contrast, the nontrivial $\left(q_{x z}\right)_{\text {loc }}=S / 2(4 \delta c \sin \theta-4 \epsilon a \cos \theta)$ decreases in magnitude as $\theta$ increases. The numerical values for the magnitudes of toroidization $T_{y}^{\text {loc }}$ (toroidal moment per unit volume $V$ ) and quadrupolization $Q_{x z}^{\text {loc }}$ are equal at $\theta=0^{\circ}$, and the value is $3.3 \times 10^{-2} \mu_{B} / \AA^{2}$ (using $S=2 \mu_{B}$, $\delta=0.488, \epsilon=0.225, c / a=0.466, a=10.032 \AA$, and $V=$ $274.76 \AA^{3}$ ).

The corresponding changes in the antisymmetric part of the Compton profile are depicted in Fig. 4(a). We see that, with increase in $\theta$, the height of the first peak (the peak at smaller $\left.\left|p_{y}\right|\right)$ in $J^{a}\left(p_{y}\right)$ increases, whereas the second peak (the peak at larger $\left.\left|p_{y}\right|\right)$ decreases. Correlating this behavior with the simultaneous increase in $t_{y}$ and decrease in $q_{x z}$ calculated above suggests that the two peaks may be associated with the two different ME multipoles $t_{y}$ and $q_{x z}$, respectively.

We can, further, manipulate the magnetic structure of $\mathrm{LiNiPO}_{4}$ by completely changing the spin configuration so that the symmetry changes from the actual magnetic-groundstate symmetry of Pnm'a. The introduction of new magnetic symmetries enables different ferro-type ordered ME multipoles as also can be understood from the direct dependence of the multipoles on the local magnetic dipole moments [see, e.g., the loc contribution in Eq. (5)], offering the possibility of studying the effects of ME multipoles other than $t_{y}$ and $q_{x z}$ on the antisymmetric Compton profile.

We made all three possible changes in the magnetic structure that are allowed by the structural Pnma symmetry of $\mathrm{LiNiPO}_{4}$ and also have a net ME multipole moment, while keeping the dimension of the magnetic unit cell the same as that of the structural unit cell. In the first case, we kept the $\mathrm{Ni}$ spin moments in the same $x-z$ plane but changed their relative magnetic arrangement so that the $x$ components of $\mathrm{Ni}(1)$ and $\mathrm{Ni}(4)$ are parallel to each other and antiparallel to those of $\mathrm{Ni}(2)$ and $\mathrm{Ni}(3)$, while the parallel $z$ components of $\mathrm{Ni}(1)$ and $\mathrm{Ni}(2)$ are antiparallel to those of $\mathrm{Ni}(3)$ and $\mathrm{Ni}(4)$. This cor- responds to the magnetic space group $P n^{\prime} m^{\prime} a^{\prime}$ [see Fig. 5(a)] and the IR representation $A_{u}^{-}$in Table I, which allows for a ME monopole $a$ and the quadrupole moments $q_{x^{2}-y^{2}}$ and $q_{z^{2}}$, with the momentum-space basis function $k_{x} k_{y} k_{z}$. In this case, the computed Compton profile has an antisymmetric part only along the $p_{x y z}$ direction, as shown in Fig. 4(b).

We can, further, rotate the spins in the out-of-plane direction, in such a way that they have only $y$ components. Among the various possibilities, only two arrangements support a net ME multipole moment and are allowed by symmetry. These two magnetic configurations are shown in Figs. 5(b) and 5(c). In one of these two configurations, the parallel spin moments on $\mathrm{Ni}(1)$ and $\mathrm{Ni}(4)$ are antiparallel to those of $\mathrm{Ni}(2)$ and $\mathrm{Ni}(3)$, and in the other configuration, $\mathrm{Ni}(1)$ and $\mathrm{Ni}(2)$ have parallel spins, which are antiparallel to those of $\mathrm{Ni}(3)$ and $\mathrm{Ni}(4)$. The former corresponds to the magnetic space group $P n m a^{\prime}$ and the IR representation $B_{1 u}^{-}$, while the latter corresponds to the $P n^{\prime} m a$ magnetic space group with the IR representation $B_{3 u}^{-}$. Calculations for these two magnetic structures show the presence of antisymmetric Compton profiles along $p_{z}$ and $p_{x}$, respectively [see Figs. 4(c) and 4(d)], consistent with the momentum-space basis functions for the allowed multipoles $\left\{t_{z}, q_{x y}\right\}$ and $\left\{t_{x}, q_{y z}\right\}$, respectively (see Table I).

The above analysis of the manipulation of the magnetic structure provides two important conclusions. First, it confirms our argument that all the ME multipoles contribute to the antisymmetric Compton profile and not just the toroidal moment, which is one of the central results of this paper. Secondly, it emphasizes the dependence of the antisymmetric Compton profile on the fine details of the magnetic structure,
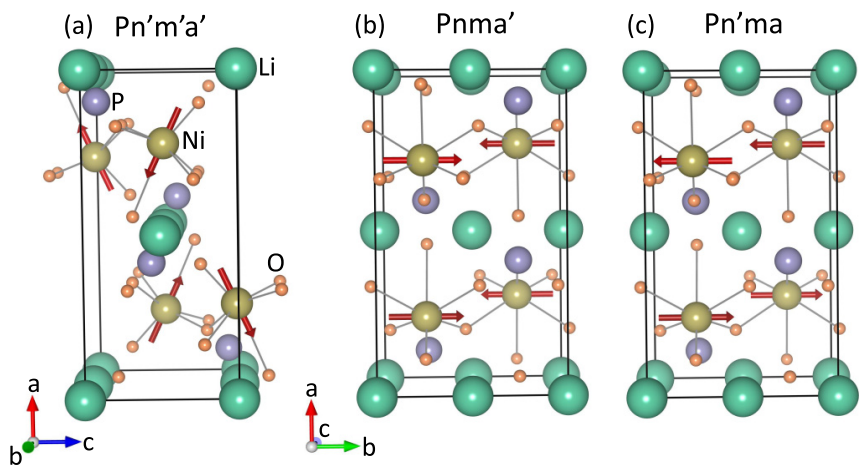

FIG. 5. Schematic illustrations of the various symmetry-allowed magnetic structures corresponding to the magnetic space groups (a) $P n^{\prime} m^{\prime} a^{\prime}$, (b) $P n m a^{\prime}$, and (c) $P n^{\prime} m a$. The arrows indicate the direction of the spin moments at the $\mathrm{Ni}$ atoms. 
TABLE III. The basis functions of the ME multipoles for the $D_{4 h}$ point group. The symbols have the same meaning as in Table I.

\begin{tabular}{lccc}
\hline \hline IR & & & Basis \\
\hline$A_{1 u}^{-}$ & ME multipole & Real space & $k$ space \\
& $a$, & $x m_{x}+y m_{y}+z m_{z}$, & $k_{x} k_{y} k_{z}\left(k_{x}^{2}-k_{y}^{2}\right)$ \\
$A_{2 u}^{-}$ & $q_{z^{2}}$ & $\left(2 z m_{z}-x m_{x}-y m_{y}\right)$ & $k_{z}$ \\
$B_{1 u}^{-}$ & $t_{z}$ & $\left(x m_{y}-y m_{x}\right)$ & $k_{x} k_{y} k_{z}$ \\
$B_{2 u}^{-}$ & $q_{x^{2}-y^{2}}$ & $\left(x m_{x}-y m_{y}\right)$ & $k_{z}\left(k_{x}^{2}-k_{y}^{2}\right)$ \\
$E_{u}^{-}$ & $q_{x y}$ & $\left(x m_{y}+y m_{x}\right)$ & $\left\{k_{x}, k_{y}\right\}$ \\
& $\left\{q_{y z}, q_{x z}\right\}$, & $\left\{\left(y m_{z}+z m_{y}\right),\left(z m_{x}+x m_{z}\right)\right\}$ & $\left\{\left(y m_{z}-z m_{y}\right),\left(z m_{x}-x m_{z}\right)\right\}$ \\
\hline \hline
\end{tabular}

suggesting that the manipulation of the spin configuration may also be used to decompose the profile into individual $\mathrm{ME}$ multipole moment contributions.

\section{B. Metallic system: $\mathbf{M n}_{2} \mathrm{Au}$}

We now turn to our second example, $\mathrm{Mn}_{2} \mathrm{Au}$, which crystallizes in the tetragonal structure $(I 4 / \mathrm{mmm})$ with point group symmetry $D_{4 h}$ [39]. The material exhibits AFM ordering with the spins having easy $a-b$ plane anisotropy [27]. In contrast to the previously discussed $\mathrm{LiNiPO}_{4}, \mathrm{Mn}_{2} \mathrm{Au}$ is a good conductor. Interestingly, the electric current in $\mathrm{Mn}_{2} \mathrm{Au}$ induces a switching of the Néel vector, which can be used to write (store) or read out information, making it a promising material for the present-day hot topic of AFM spintronics [27,28]. The ground-state magnetic structure of $\mathrm{Mn}_{2} \mathrm{Au}$, corresponding to the magnetic space group $F m^{\prime} m m$ with the [110] Néel vector, is depicted in Fig. 6(a). Similarly to $\mathrm{LiNiPO}_{4}$, the magnetic structure in $\mathrm{Mn}_{2} \mathrm{Au}$ breaks the inversion symmetry, allowing for ME toroidal and quadrupole moments leading to an offdiagonal $\mathcal{M}_{i j}$ tensor. The specific magnetic configuration of $\mathrm{Mn}_{2} \mathrm{Au}$ leads to interesting symmetry properties between the allowed ME multipoles, which, further, manifest in the asymmetric band structure as well as in the antisymmetric Compton profile in $\mathrm{Mn}_{2} \mathrm{Au}$. Below we discuss these symmetry relations in detail and point out the intriguing features of the band structure and the antisymmetric profile in $\mathrm{Mn}_{2} \mathrm{Au}$, which in addition to their relevance for Compton scattering might also be important for other experiments.

\section{Symmetry analysis}

The allowed ME multipoles and their real- and momentumspace basis functions in the different IR representations of the point group $D_{4 h}$, relevant to $\mathrm{Mn}_{2} \mathrm{Au}$, are listed in Table III. The magnetic structure of $\mathrm{Mn}_{2} \mathrm{Au}$ belongs to the IR representation $E_{u}^{-}$, which allows for toroidal moments along the $x$ and $y$ directions, $t_{x}$ and $t_{y}$, and also for quadrupole moments $q_{y z}$ and $q_{x z}$. No additional local ME multipoles are allowed at the Mn site, forbidding any additional ME multipoles with antiferro-type arrangement. It is interesting to point out here that the allowed multipole tensor for the magnetic ground state, corresponding to the magnetic space group $F m^{\prime} m m$, has the constraints $\mathcal{M}_{x z}=\mathcal{M}_{y z}$, and $\mathcal{M}_{z x}=\mathcal{M}_{z y}$. This, in turn, imposes an additional constraint on the allowed ME multipoles, that $t_{x}+t_{y}=q_{y z}-q_{x z}$ [see Eq. (1)].

As expected, the momentum-space basis functions of the time-odd, parity-odd ME multipoles in Table III are always spin independent and of odd order in $k$. According to the momentum-space basis function for the allowed ME multipoles in $\mathrm{Mn}_{2} \mathrm{Au}$, antisymmetric Compton profiles should appear along the $k_{x}$ and $k_{y}$ directions (see Table III), which we, further, verify by explicitly computing the antisymmetric Compton profile as discussed below.

\section{DFT results for the Compton profile and ME multipoles}

We compute the antisymmetric Compton profile for $\mathrm{Mn}_{2} \mathrm{Au}$ using the ELK code [35,36], following the same method as discussed in Sec. III A 2. All calculations are performed with the experimentally reported structure [39] within the $\mathrm{LDA}+\mathrm{SOC}+U$ formalism with the correlation parameters $U=1 \mathrm{eV}$ and $J=0.5 \mathrm{eV}$ at the $\mathrm{Mn}$ site. The specific choice of the correlation parameters is guided by recent photoemission measurements [40], the results of which can be reproduced satisfactorily with these parameters. It is important to point out here that, in contrast to the insulating $\mathrm{LiNiPO}_{4}$, the antisymmetric Compton profile in $\mathrm{Mn}_{2} \mathrm{Au}$ has a strong dependence on the choice of $k$-point mesh, and the convergence of the antisymmetric profile is achieved at a much higher $k$-point grid of $31 \times 31 \times 12$.

In the computed magnetic ground state, the magnetic moment at each Mn site projected onto an ionic radius 2.4 a.u. is about $4.1 \mu_{B}$. In this magnetic structure, our calculations find that only the $t_{x}, t_{y}, q_{x z}$, and $q_{y z}$ ME multipoles have nonzero values and that they have ferro-type arrangements between different $\mathrm{Mn}$ atoms, as expected from the symmetry analysis. In addition, the computed components of the toroidal moment as well as the quadrupole moments are not independent but are related to each other by $t_{x}=-t_{y}$ and $q_{x z}=q_{y z}$. These relations satisfy the symmetry-imposed condition on the $\mathrm{ME}$ multipoles $t_{x}+t_{y}=q_{y z}-q_{x z}$, as mentioned earlier.

Interestingly, for the [110] Néel vector orientation, which is symmetrically equivalent to the [110] Néel vector but represents a different magnetoelectric domain [40,41], the relative signs of the toroidal and the quadrupole moment components switch, such that $t_{x}=t_{y}$ and $q_{x z}=-q_{y z}$. These relative orientations of the computed multipoles can be understood directly from their formal definitions, given in Sec. II. For example, according to the definition of the toroidal moment, $t_{x}=\frac{1}{2}\left(y m_{z}-z m_{y}\right)$ and $t_{y}=\frac{1}{2}\left(x m_{z}-z m_{x}\right)$, which gives $t_{x}=$ $-t_{y}$ for $m_{x}=m_{y}$ and $m_{z}=0$ ([110] Néel vector), but $t_{x}=t_{y}$ for $m_{x}=-m_{y}$ and $m_{z}=0$ ([110] Néel vector). Similarly, it is easy to show that $q_{x z}$ and $q_{y z}$ are the same in both sign and magnitude for the [110] orientation of the Néel vector, 


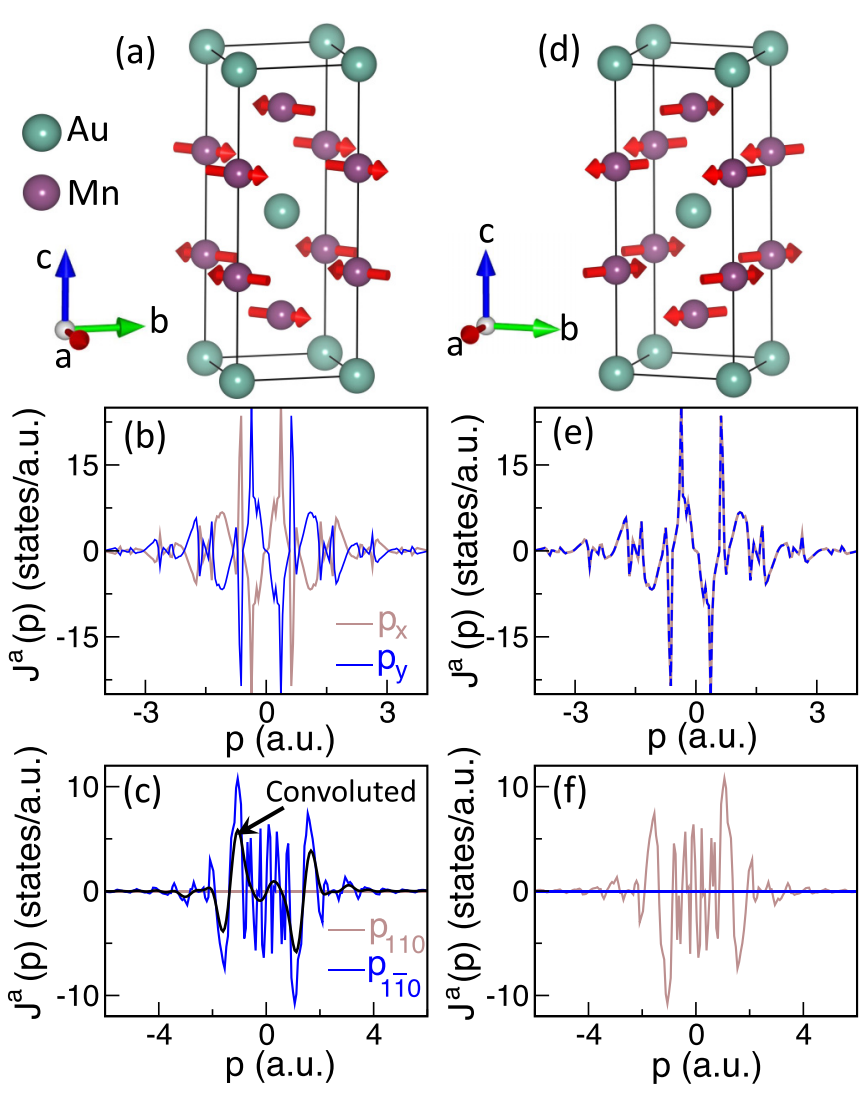

FIG. 6. Antisymmetric Compton profile in $\mathrm{Mn}_{2} \mathrm{Au}$. (a) The crystal and magnetic structures of the ground state of $\mathrm{Mn}_{2} \mathrm{Au}$ corresponding to the Néel vector [110]. Arrows indicate the direction of the spin magnetic moments at the Mn sites. (b) The antisymmetric parts $\left(J^{a}\right)$ of the Compton profile along $p_{x}$ and $p_{y}$, indicating equal and opposite contributions along these directions. This results in an asymmetry along [1시 but an absence of asymmetry along [110]. (c) The computed antisymmetric Compton scattering profiles along the [110] and [110] directions reflect the presence and absence of asymmetry, respectively. The convolution of the computed antisymmetric profile along the $p_{1 \overline{1} 0}$ direction with a Gaussian function of 0.44 a.u. FWHM is also shown. (d) The magnetic structure for Néel vector [110]. The corresponding antisymmetric Compton profile along (e) $p_{x}$ and $p_{y}$ and (f) $p_{110}$ and $p_{1 \overline{1} 0}$. In contrast to the Néel vector [110], here the antisymmetric profiles along $p_{x}$ and $p_{y}$ are the same in both magnitude and sign, leading to a vanishing antisymmetric profile along $p_{1 \overline{1} 0}$ but a nonzero $J^{a}$ along the [110] direction. All profiles are normalized, so that the total profile is normalized to 63 electrons, which is the total number of valence electrons per formula unit of $\mathrm{Mn}_{2} \mathrm{Au}$ in the calculation. The antisymmetric part of the Compton profile is magnified by a factor of $10^{3}$.

while they have opposite signs when the Néel vector is along [110]. The computed magnitudes of the independent components of the allowed ME multipoles at the Mn site are $\tilde{t}_{y}=-5.3 \times 10^{-3} \mu_{B}$ and $\tilde{q}_{x z}=-6.2 \times 10^{-3} \mu_{B}$, where we have added the $p-d$ and $p-s$ matrix element contributions to obtain the total atomic-site contribution for each Mn atom. These ME multipoles lead to interesting consequences in the corresponding band structure and the antisymmetric Compton profile as we now proceed to discuss.
The calculation of the Compton profile along the three Cartesian directions $x, y$, and $z$ shows an antisymmetric component only along $p_{x}$ and $p_{y}$, while $J^{a}$ is absent along $p_{z}$, in agreement with the symmetry analysis, discussed earlier. As seen from Figs. 6(b) and 6(c), unlike $\mathrm{LiNiPO}_{4}$, the antisymmetric profile in $\mathrm{Mn}_{2} \mathrm{Au}$ has sharp peaks at lower momentum, which also change their signs rapidly. Such oscillating behavior may be ascribed to the fine details of the Fermi surface hosting numerous, strongly dispersed electronic bands near the Fermi energy of the metallic system, $\mathrm{Mn}_{2} \mathrm{Au}$. Also, the antisymmetric profile has a larger magnitude compared with that in $\mathrm{LiNiPO}_{4}$, which may be attributed to the presence of the heavy $\mathrm{Au}$ atom leading to stronger SOC effects in $\mathrm{Mn}_{2} \mathrm{Au}$.

It is interesting to point out here that the anti-symmetric parts along the $p_{x}$ and $p_{y}$ directions are exactly equal and opposite to each other, i.e., $J^{a}\left(p_{x}\right)=-J^{a}\left(p_{y}\right)$ [see Fig. 6(b)]. This leads to a vanishing antisymmetric Compton profile along the [110] direction in momentum space, while a nonzero antisymmetric profile appears in the perpendicular direction [1110] as shown in Fig. 6(c). The convolution of the computed profile with an experimental resolution [Gaussian of full width at half maximum (FWHM)] of 0.44 a.u. shows that while the sharp peaks as well as their oscillations in the low-momentum regime may be suppressed, some oscillating features can still be captured within this resolution limit [see Fig. 6(c)].

Furthermore, switching of the Néel vector to the [1110] direction results in an exactly opposite situation, as depicted in Figs. 6(e) and 6(f). In this case, the antisymmetric profiles along $p_{x}$ and $p_{y}$ are equal in both magnitude and sign, leading to the presence of an antisymmetric Compton profile along the [110] direction in momentum space, while it is absent along [1 $1 \overline{1} 0]$. This may be of importance to the experimental measurements, where an electric-current-induced switching of the Néel vector, in turn, results in a switching of the antisymmetric Compton profile from the [110] to the [110] direction in the momentum space.

These distinct features of the antisymmetric Compton profile in $\mathrm{Mn}_{2} \mathrm{Au}$ may be understood from careful analysis of the corresponding band structures. The computed band structure for the magnetic ground state of $\mathrm{Mn}_{2} \mathrm{Au}$ with [110] Néel vector shows that the bands are asymmetric along the $k_{x}$ and $k_{y}$ directions [see Figs. 7(a) and 7(b)]. The asymmetry of the bands in the presence of SOC follows from similar symmetry arguments to those discussed above for the case of $\mathrm{LiNiPO}_{4}$. Interestingly, the comparison of the bands along $k_{x}$ and $k_{y}$ shows that the band asymmetry is opposite for these two directions, explaining the opposite signs of the computed antisymmetric profiles along $p_{x}$ and $p_{y}$. The asymmetry of the bands is even more pronounced along the [110] direction of the momentum space, while along the [110] direction the bands are absolutely symmetric as depicted in Figs. 7(c) and 7(d). This explains, further, the presence and the absence of the antisymmetric Compton profile along the [110] and [110] directions, respectively.

In contrast, switching of the magnetization direction to [11ī0] results in similar asymmetries along the $k_{x}$ and $k_{y}$ directions as understood by comparing the bands shown in Figs. 7(e) and 7(f). This results in a band asymmetry along the [110] direction, while the bands remain symmetric in the perpendicular [1 $1 \overline{1} 0$ ] direction [see Figs. $7(\mathrm{~g})$ and 7(h)], 



FIG. 7. The asymmetry in the band structure of $\mathrm{Mn}_{2} \mathrm{Au}$, induced by the ME multipoles, for the Néel vectors [110] [(a)-(d)] and [110] [(e)-(h)]. The band structure along the (a) $k_{x}$, (b) $k_{y}$, (c) [110], and (d) [110] directions in the momentum space for the Néel vector [110] with the corresponding magnetic structure shown in Fig. 6(a). Comparison of (a) and (b) shows that the asymmetries of the bands are opposite along $k_{x}$ and $k_{y}$ for the Néel vector [110]. This results in an absence of asymmetry along the [110] direction in (c), while the asymmetry along the [1 $\overline{1} 0]$ direction is clearly visible in (d). Comparison with bands in the absence of SOC shows that the asymmetry is present only in the presence of SOC. Doubling the strength of the SOC further enhances this asymmetry, indicating the importance of SOC. (e)-(h) The same as in (a)-(d) but for the Néel vector [11̄0]. The corresponding magnetic structure is in Fig. 6(d). In this case, bands along (e) $k_{x}$ and (f) $k_{y}$ have the same asymmetry, leading to (g) asymmetric bands along the [110] direction and (h) absence of asymmetry along the [110] direction in the momentum space.

consistent with the switching of the antisymmetric Compton profile by changing the orientation of the Néel vector in $\mathrm{Mn}_{2} \mathrm{Au}$. Interestingly, changes in the energy of the conduction electrons corresponding to the different Néel vectors have also been recently reported in photoemission measurements on $\mathrm{Mn}_{2} \mathrm{Au}$ thin films [40]. (Note that the unit cell of the reported thin film in Ref. [40] is rotated with respect to the bulk unit cell considered in our calculation, and our [1ㅣㅣ and [110] directions correspond to their $k_{x}, k_{y}$ directions, respectively.) The confirmation of left-right asymmetry in the band structure is, however, not possible from these measurements as the photoemission intensity from the two domains was averaged out [40].

Similarly to the case of $\mathrm{LiNiPO}_{4}$, these asymmetries in the band structure can be traced back to the allowed ME multipoles in momentum space (see Table III). Physically speaking, the spin asymmetry, designated by the allowed ME multipoles, couples to the momentum space via SOC, resulting in a band asymmetry. This is further evidenced by comparing the band structures for two different values of SOC, as shown in Fig. 7(d), where we see that doubling the strength of the SOC results in an enhanced asymmetry.
Finally, we want to emphasize the one-to-one correspondence between the band asymmetries along the [110] or [110] directions in the momentum space for the two Néel vectors [110] and [1 10$]$ in $\mathrm{Mn}_{2} \mathrm{Au}$ and the respective directions of the allowed toroidal moment vectors $\vec{t}=t_{y}(\hat{i}-\hat{j})$ or $\vec{t}=t_{y}(\hat{i}+\hat{j})$. We note that the components of the quadrupole moment tensors $q_{x z}, q_{y z}$ have also the same symmetry as the allowed toroidal moment, belonging to the same $E_{u}^{-}$irreducible representation. It is interesting to point out that the same ME multipoles can provide a convenient description of the current-induced switching of the Néel vectors in $\mathrm{Mn}_{2} \mathrm{Au}$ [29]. Our theoretical analysis suggests a presence of band asymmetry in $\mathrm{Mn}_{2} \mathrm{Au}$ and its switching with the change in Néel vector, which are signatures of the allowed ME multipole moments in the system.

\section{SUMMARY AND OUTLOOK}

In summary, we have presented a comprehensive theoretical analysis of the antisymmetric Compton profile in materials with noncentrosymmetric magnetic ordering. We have shown that the antisymmetric Compton profile is a signature of parity-odd, time-odd ME multipoles. The ME multipoles, which break both space-inversion and time-reversal symmetries, generate an asymmetry in the magnetization density, the details of which are governed by the specific components of the ME multipoles that occur. The asymmetry, in turn, can be characterized by the basis functions of the corresponding ME multipoles, which, since the ME multipoles are of odd parity, are different in real and momentum space. In particular, while the real-space basis functions for ME multipoles are always spin dependent, the momentum-space basis functions are not. As a result, they can be probed in $k$ space without any spin-resolved measurements. In addition, since the momentum-space bases for ME multipoles are always of odd order in $k$, they generate asymmetry in the electronic band dispersion in momentum space, with the direction of asymmetry determined by the specific momentum-space basis. Since the Compton scattering is a measure of the electron-momentum density, an asymmetry in the Compton scattering profile is generated in the presence of ME multipoles. The coupling of the magnetization asymmetry to the space requires the presence of SOC, indicating the importance of SOC effects in generating the antisymmetric Compton profile.

In addition to emphasizing the role of the real-spacemomentum-space duality in understanding the antisymmetric profile, our work points out the two essential ingredients for an antisymmetric Compton profile: time-odd, parity-odd $\mathrm{ME}$ multipoles and SOC. This can in turn be used to guide the search for suitable candidate materials with larger antisymmetric Compton profiles. We note that earlier attempts were not inconsistent with the theoretical predictions, but were not conclusive as the observed antisymmetric signal fell within the statistical error [21,22]. The ME multipoles requirement means that the antisymmetric profile has a strong dependence on the magnetic structure of the system. This leads to the possibility of tuning the profile by manipulating the magnetic structure, as discussed in Sec. III A 3. The SOC requirement suggests a route to identifying materials with a stronger effect by choosing materials with heavy elements, and therefore 
strong SOC. Furthermore, the finding of this paper that the antisymmetric Compton profile is present for all ME materials, and not just those with ME toroidal moment, also broadens the scope for candidate materials.

Finally, our theoretical studies on the representative insulating and metallic candidate materials indicate some distinct features in the corresponding antisymmetric Compton profiles. While the antisymmetric profile has broad peaks in insulating $\mathrm{LiNiPO}_{4}$, the peaks are rather sharp and oscillating, especially in the low-momentum regime, for the metallic system $\mathrm{Mn}_{2} \mathrm{Au}$. We also predict some detailed features that should be experimentally observable, for example, opposite changes in the two peak heights in $\mathrm{LiNiPO}_{4}$ as the spin canting angle is varied, and may give further insight into the detailed role of the ME multipoles. Of particular interest is the antisymmetric Compton profile in $\mathrm{Mn}_{2} \mathrm{Au}$, the direction of which can be switched between $p_{1 \overline{1} 0}$ and $p_{110}$ by changing the orientation of the Mn spin moments from [110] to [110]. We hope that our predictions will stimulate more experiments in this direction.

\section{ACKNOWLEDGMENTS}

The authors thank Stephen Collins, Jon Duffy, Michael Fechner, and Urs Staub for stimulating discussions. This work was supported by the European Research Council (ERC) under the European Union's Horizon 2020 Research and Innovation Programme project HERO Grant Agreement No. 810451. Computational resources were provided by ETH Zürich (Euler cluster).
[1] L. D. Landau and E. M. Lifshitz, The Classical Theory of Fields, 4th ed. (Butterworth-Heinemann, Oxford, 1980).

[2] J. D. Jackson, Classical Electrodynamics, 3rd ed. (Wiley, New York, 1999).

[3] R. J. Blin-Stoyle, Theories of nuclear moments, Rev. Mod. Phys. 28, 75 (1956).

[4] R. E. Raab and O. L. D. Lange, Multipole Theory in Electromagnetism: Classical, Quantum, and Symmetry Aspects, with Applications (Oxford University Press, Oxford, 2004).

[5] T. Kaelberer, V. A. Fedotov, N. Papasimakis, D. P. Tsai, and N. I. Zheludev, Toroidal dipolar response in a metamaterial, Science 330, 1510 (2010).

[6] N. A. Spaldin, M. Fiebig, and M. Mostovoy, The toroidal moment in condensed-matter physics and its relation to the magnetoelectric effect, J. Phys.: Condens. Matter 20, 434203 (2008).

[7] S. Hayami, M. Yatsushiro, Y. Yanagi, and H. Kusunose, Classification of atomic-scale multipoles under crystallographic point groups and application to linear response tensors, Phys. Rev. B 98, 165110 (2018).

[8] N. A. Spaldin, M. Fechner, E. Bousquet, A. Balatsky, and L. Nordström, Monopole-based formalism for the diagonal magnetoelectric response, Phys. Rev. B 88, 094429 (2013).

[9] H. Watanabe and Y. Yanase, Nonlinear electric transport in oddparity magnetic multipole systems: Application to Mn-based compounds, Phys. Rev. Research 2, 043081 (2020).

[10] V. Dubovik and V. Tugushev, Toroid moments in electrodynamics and solid-state physics, Phys. Rep. 187, 145 (1990).

[11] T. Onimaru, K. T. Matsumoto, Y. F. Inoue, K. Umeo, T. Sakakibara, Y. Karaki, M. Kubota, and T. Takabatake, Antiferroquadrupolar Ordering in a Pr-Based Superconductor $\operatorname{PrIr}_{2} \mathrm{Zn}_{20}$, Phys. Rev. Lett. 106, 177001 (2011).

[12] F. Cricchio, F. Bultmark, O. Grånäs, and L. Nordström, Itinerant Magnetic Multipole Moments of Rank Five as the Hidden Order in $\mathrm{URu}_{2} \mathrm{Si}_{2}$, Phys. Rev. Lett. 103, 107202 (2009).

[13] P. Santini, S. Carretta, G. Amoretti, R. Caciuffo, N. Magnani, and G. H. Lander, Multipolar interactions in $f$-electron systems: The paradigm of actinide dioxides, Rev. Mod. Phys. 81, 807 (2009).
[14] S. Sumita, T. Nomoto, and Y. Yanase, Multipole Superconductivity in Nonsymmorphic $\mathrm{Sr}_{2} \mathrm{IrO}_{4}$, Phys. Rev. Lett. 119, 027001 (2017).

[15] H. Watanabe and Y. Yanase, Magnetic hexadecapole order and magnetopiezoelectric metal state in $\mathrm{Ba}_{1-x} \mathrm{~K}_{x} \mathrm{Mn}_{2} \mathrm{As}_{2}$, Phys. Rev. B 96, 064432 (2017).

[16] L. Fu, Parity-Breaking Phases of Spin-Orbit-Coupled Metals with Gyrotropic, Ferroelectric, and Multipolar Orders, Phys. Rev. Lett. 115, 026401 (2015).

[17] U. Staub, Y. Bodenthin, C. Piamonteze, M. García-Fernández, V. Scagnoli, M. Garganourakis, S. Koohpayeh, D. Fort, and S. W. Lovesey, Parity- and time-odd atomic multipoles in magnetoelectric $\mathrm{GaFeO}_{3}$ as seen via soft x-ray Bragg diffraction, Phys. Rev. B 80, 140410(R) (2009).

[18] K. Kimura, T. Katsuyoshi, Y. Sawada, S. Kimura, and T. Kimura, Imaging switchable magnetoelectric quadrupole domains via nonreciprocal linear dichroism, Commun. Mater. 1, 39 (2020).

[19] S. W. Lovesey, Magneto-electric operators in neutron scattering from electrons, J. Phys.: Condens. Matter 26, 356001 (2014).

[20] S. W. Lovesey, T. Chatterji, A. Stunault, D. D. Khalyavin, and G. van der Laan, Direct Observation of Anapoles by Neutron Diffraction, Phys. Rev. Lett. 122, 047203 (2019).

[21] S. P. Collins, D. Laundy, T. Connolley, G. van der Laan, F. Fabrizi, O. Janssen, M. J. Cooper, H. Ebert, and S. Mankovsky, On the possibility of using X-ray Compton scattering to study magneto-electrical properties of crystals, Acta Crystallogr., Sect. A: Found. Adv. 72, 197 (2016).

[22] S. Bhowal, D. O’Neill, N. A. Spaldin, U. Staub, J. Duffy, and S. P. Collins, Anti-symmetric Compton scattering in $\mathrm{LiNiPO}_{4}$ : Towards a direct probe of the magneto-electric multipole moment, arXiv:2106.02431.

[23] C. Ederer and N. A. Spaldin, Towards a microscopic theory of toroidal moments in bulk periodic crystals, Phys. Rev. B 76, 214404 (2007).

[24] H. Watanabe and Y. Yanase, Group-theoretical classification of multipole order: Emergent responses and candidate materials, Phys. Rev. B 98, 245129 (2018).

[25] M. Mercier and P. Bauer, Magnetoelectric Effects in $\mathrm{LiNiPO}_{4}$, C. R. Acad. Sci. Paris 267, 465 (1968). 
[26] A. B. Shick, S. Khmelevskyi, O. N. Mryasov, J. Wunderlich, and T. Jungwirth, Spin-orbit coupling induced anisotropy effects in bimetallic antiferromagnets: A route towards antiferromagnetic spintronics, Phys. Rev. B 81, 212409 (2010).

[27] S. Y. Bodnar, L. Šmejkal, I. Turek, T. Jungwirth, O. Gomonay, J. Sinova, A. A. Sapozhnik, H. J. Elmers, M. Kläui, and M. Jourdan, Writing and reading antiferromagnetic $\mathrm{Mn}_{2} \mathrm{Au}$ by Néel spin-orbit torques and large anisotropic magnetoresistance, Nat. Commun. 9, 348 (2018).

[28] X. Chen, X. Zhou, R. Cheng, C. Song, J. Zhang, Y. Wu, Y. Ba, H. Li, Y. Sun, Y. You, Y. Zhao, and F. Pan, Electric field control of Néel spin-orbit torque in an antiferromagnet, Nat. Mater. 18, 931 (2019).

[29] F. Thöle, A. Keliri, and N. A. Spaldin, Concepts from the linear magnetoelectric effect that might be useful for antiferromagnetic spintronics, J. Appl. Phys. (Melville, NY) 127, 213905 (2020).

[30] I. Abrahams and K. S. Easson, Structure of lithium nickel phosphate, Acta Crystallogr., Sect. C: Cryst. Struct. Commun. 49, 925 (1993).

[31] E. Fogh, T. Kihara, R. Toft-Petersen, M. Bartkowiak, Y. Narumi, O. Prokhnenko, A. Miyake, M. Tokunaga, K. Oikawa, M. K. Sørensen, J. C. Dyrnum, H. Grimmer, H. Nojiri, and N. B. Christensen, Magnetic structures and quadratic magnetoelectric effect in $\mathrm{LiNiPO}_{4}$ beyond 30 T, Phys. Rev. B 101, 024403 (2020).

[32] T. B. S. Jensen, N. B. Christensen, M. Kenzelmann, H. M. Rønnow, C. Niedermayer, N. H. Andersen, K. Lefmann, J. Schefer, M. v. Zimmermann, J. Li, J. L. Zarestky, and D. Vaknin, Field-induced magnetic phases and electric polarization in $\mathrm{LiNiPO}_{4}$, Phys. Rev. B 79, 092412 (2009).

[33] T. Inui, Y. Tanabe, and Y. Onodera, Group Theory and Its Applications in Physics, Springer Series in Solid-State Sciences Vol. 78 (Springer, New York, 1990).
[34] J. Perez-Mato, S. Gallego, E. Tasci, L. Elcoro, G. de la Flor, and M. Aroyo, Symmetry-based computational tools for magnetic crystallography, Annu. Rev. Mater. Res. 45, 217 (2015).

[35] The Elk Code, http://elk.sourceforge.net/.

[36] D. Ernsting, D. Billington, T. D. Haynes, T. E. Millichamp, J. W. Taylor, J. A. Duffy, S. R. Giblin, J. K. Dewhurst, and S. B. Dugdale, Calculating electron momentum densities and Compton profiles using the linear tetrahedron method, J. Phys.: Condens. Matter 26, 495501 (2014).

[37] See Supplemental Material at http://link.aps.org/supplemental/ 10.1103/PhysRevResearch.3.033185 for the data files containing also the valence contribution to the Compton profile; for more details, refer to https://archive.materialscloud.org/record/ 2021.117.

[38] F. Biggs, L. Mendelsohn, and J. Mann, Hartree-Fock Compton profiles for the elements, At. Data Nucl. Data Tables 16, 201 (1975).

[39] P. Wells and J. H. Smith, The structure of $\mathrm{Mn}_{2} \mathrm{Au}$ and $\mathrm{Mn}_{3} \mathrm{Au}$, Acta Crystallogr., Sect. A: Cryst. Phys., Diffr., Theor. Gen. Crystallogr. 26, 379 (1970).

[40] H. J. Elmers, S. V. Chernov, S. W. D'Souza, S. P. Bommanaboyena, S. Y. Bodnar, K. Medjanik, S. Babenkov, O. Fedchenko, D. Vasilyev, S. Y. Agustsson, C. Schlueter, A. Gloskovskii, Y. Matveyev, V. N. Strocov, Y. Skourski, L. Šmejkal, J. Sinova, J. Minár, M. Kläui, G. Schönhense et al., Néel Vector Induced Manipulation of Valence States in the Collinear Antiferromagnet $\mathrm{Mn}_{2} \mathrm{Au}$, ACS Nano 14, 17554 (2020).

[41] A. A. Sapozhnik, M. Filianina, S. Y. Bodnar, A. Lamirand, M.-A. Mawass, Y. Skourski, H.-J. Elmers, H. Zabel, M. Kläui, and M. Jourdan, Direct imaging of antiferromagnetic domains in $\mathrm{Mn}_{2} \mathrm{Au}$ manipulated by high magnetic fields, Phys. Rev. B 97, 134429 (2018). 